\title{
Targeting Hypoxia to Augment Mucosal Barrier Function
}

\author{
Caleb J. Kelly and Sean P. Colgan* \\ Mucosal Inflammation Program, Department of Medicine, University of Colorado Health Sciences Center, Aurora, \\ CO USA
}

\begin{abstract}
Sites of inflammation are associated with profound changes in tissue metabolism. Studies in vitro and in vivo have shown that the activation of the hypoxia-inducible factor (HIF) serves as an adaptive pathway for the resolution of inflammation associated with various murine disease models. The resolution of disease occurs, at least in part, through transcriptional regulation of non-classical epithelial barrier genes. There is significant recent interest in harnessing hypoxia-inducible pathways, including targeting the HIF and the proyl-hydroxylase (PHD) enzymes that stabilize HIF, to promote mucosal healing. Here, we review the signaling pathways involved and define how hypoxia-associated signaling provides mechanistic insight into augmenting barrier function in mucosal inflammatory disease.
\end{abstract}

Keywords: hypoxia, inflammation, epithelia, colitis, transcription

\section{INTRODUCTION}

Mucosal tissues, such as the bladder, lung or intestine, provide a physical barrier between biologic compartments, preventing the free mixing of luminal antigenic material with the lamina propria which houses the mucosal immune system. For this purpose, epithelia are centrally positioned for electrolyte and fluid homeostasis as well as nutrient uptake and detoxification in that they mediate vectorial and selective transport of ions, water and macromolecules between blood and the external environment [1]. The establishment and maintenance of a selectively permeable barrier occurs through interactions of the extracellular domains of multiple transmembrane adhesion domains between adjacent cells (adherens junction, tight junction, gap junction) or between the mucosal layer and extracellular matrix components (see Fig. 1). These interactions not only determine the physical integrity of the tissue as a whole, but also establish the physical organization of lipids and proteins within in the plasma membrane in a polarized fashion.

Anatomical features of the intestine provide a fascinating oxygenation profile since, even under physiologic conditions, the intestinal mucosa experiences profound fluctuations in blood flow and metabolism. For example, less than $5 \%$ of total blood volume is present in the gut during fasting, but following ingestion of a meal, approximately $30 \%$ of total blood volume is present in the gastrointestinal tract. Such changes in blood flow also result in marked shifts in local $\mathrm{pO}_{2}$. Notably, there is a steep oxygen gradient from the anaerobic lumen of the intestine across the epithelium into the highly vascularized sub-epithelium. From this perspective, it is perhaps not surprising that the epithelium has evolved a number of features to cope with these dramatic

*Address correspondence to this author at the Mucosal Inflammation Program, RC2 Room 10025, 12700 E. $19^{\text {th }}$ Ave, Aurora, CO 80045, USA. Tel: 303-724-7235; E-mail: sean.colgan@ucdenver.edu metabolic shifts. In fact, studies comparing functional responses between epithelial cells from different tissues have revealed that intestinal epithelial cells seem to be uniquely resistant to hypoxia and that an extremely low level of oxygenation within the normal intestinal epithelial barrier (so-called "physiologic hypoxia") may be a regulatory adaptation mechanism to the steep oxygen gradient [2]. Others have recently reviewed the details of physical barrier disruption in inflammation [3, 4]. Here, we will summarize recent aspects of augmenting barrier function through lessons learned studying signaling related to hypoxia and inflammation.

\section{BARRIER FUNCTION AND "INFLAMMATORY HYPOXIA"}

Sites of mucosal inflammation are characterized by profound changes in tissue metabolism, including local depletion of nutrients, imbalances in tissue oxygen supply and demand, and the generation of large quantities of reactive nitrogen and oxygen intermediates [5]. In part, these changes can be attributed to recruitment of inflammatory cells, including myeloid cells such as neutrophils (polymorphonuclear cells; PMNs) and monocytes. PMNs are recruited by chemoattractants generated at sites of active inflammation as part of the innate host immune response to microorganisms. Once at the sites of inflammation, the nutrient, energy and oxygen demands of the PMNs increase to accomplish the processes of phagocytosis and microbial killing. It has long been known that PMNs are primarily glycolytic cells, with few mitochondria and little energy produced from respiration [6]. A predominantly glycolytic metabolism ensures that PMN can function at the low oxygen concentrations (even anoxia) associated with inflammatory lesions.

Once at the inflammatory site, PMNs recognize and engulf pathogens and activate the release of antibacterial peptides, proteases and reactive oxygen species (ROS; superoxide anion, hydrogen peroxide, hydroxyl radical and hypo- 


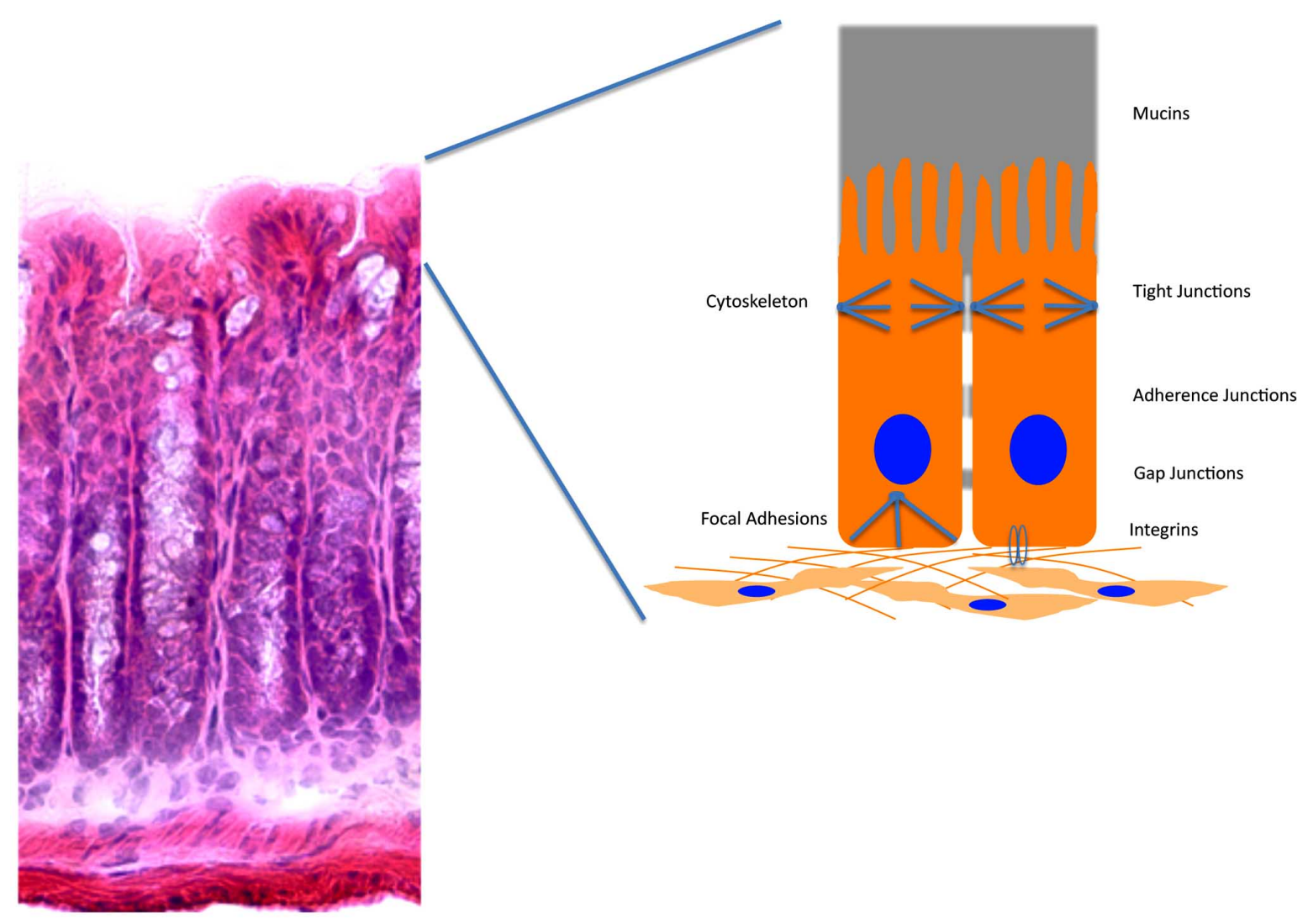

Fig. (1). Contributing components to barrier function in the intestinal mucosa. As outlined here, multiple membrane and membraneassociated components contribute to overall epithelial integrity. Each of these components is dynamically regulated during inflammation and in association with hypoxia (see text).

chlorous acid) into the vacuole, which together kill the invading microbes [7]. ROS are produced by phagocytes in a powerful oxidative burst, driven by a rapid increase in oxygen uptake and glucose consumption, which in turn triggers further generation of ROS. When activated, it is estimated that PMNs can consume up to 10 times more $\mathrm{O}_{2}$ than any other cell in the body. Notably, the PMN oxidative burst is not hindered by even relatively low $\mathrm{O}_{2}$ (as low as $4.5 \% \mathrm{O}_{2}$ ) [8], which is important, since it means that ROS can be generated in the relatively low $\mathrm{O}_{2}$ environment of inflamed intestinal mucosa [9].

Murine models of intestinal inflammation coupled with techniques to define metabolic deficits have been useful in defining the existence of hypoxia in the inflammatory setting [10]. Tissue staining with nitroimidazole dyes (see Fig. 2) has revealed two profound observations. First, in the normal intestinal epithelial cells, especially in the colon, "physiologic hypoxia" predominates. Whether such low oxygen levels function to regulate basal gene expression in intestinal epithelial cells is not known. Second, the inflammatory lesions seen in these mouse models are profoundly hypoxic or even anoxic, similar to that seen in some large tumors, and penetrate deep into the mucosal tissue. It is likely that there are multiple contributing factors, such as vasculitis, vasoconstriction, edema, and increased $\mathrm{O}_{2}$ consumption, which predispose the inflamed intestinal epithelia to decreased oxygen delivery and hypoxia [11]. These studies have allowed us to define and extend such "inflammatory hypoxia" to defining endpoints of hypoxia and development of potential therapies based on these concepts.

\section{HYPOXIA-INDUCIBLE FACTOR}

HIF is a member of the Per-ARNT-Sim family of basic helix-loop-helix transcription factors that bind hypoxia response elements (HREs) at target gene loci under hypoxic conditions [12]. Functional HIF exists as an $\alpha / \beta$ heterodimer, comprising both a constitutive subunit (HIF-1 $\beta$ ), and a hypoxia-inducible alpha component, stabilization of which is regulated in part by a family of oxygen- and iron-dependent prolyl hydroxylase (PHD) enzymes [13]. To date, three regulatory subunits have been identified, namely $\mathrm{HIF}-1 \alpha$, HIF$2 \alpha$, and HIF- $3 \alpha$ with the highest level of sequence homology conserved between HIF- $1 \alpha$ and HIF- $2 \alpha$ [14]. Evidence from genetic mouse models implies that HIF-1 and HIF-2 play non-redundant roles [14] despite their concurrent expression in many cell types, including intestinal epithelial cells [15]. Several studies have indicated that these proteins modulate the transcription of an overlapping but distinct set of target genes and those transcriptional responses may be integrated in ways that support specific adaptations to hypoxia. For instance, transcriptional regulation of target genes encoding glycolytic enzymes appears to be more discretely coordinated by HIF-1 than HIF-2 [16], whereas HIF-2 selectively regulates gene expression of factors involved in duodenal iron homeostasis [17, 18] and in early erythropoiesis [18]. The N-terminal transactivation domain is proposed to medi- 


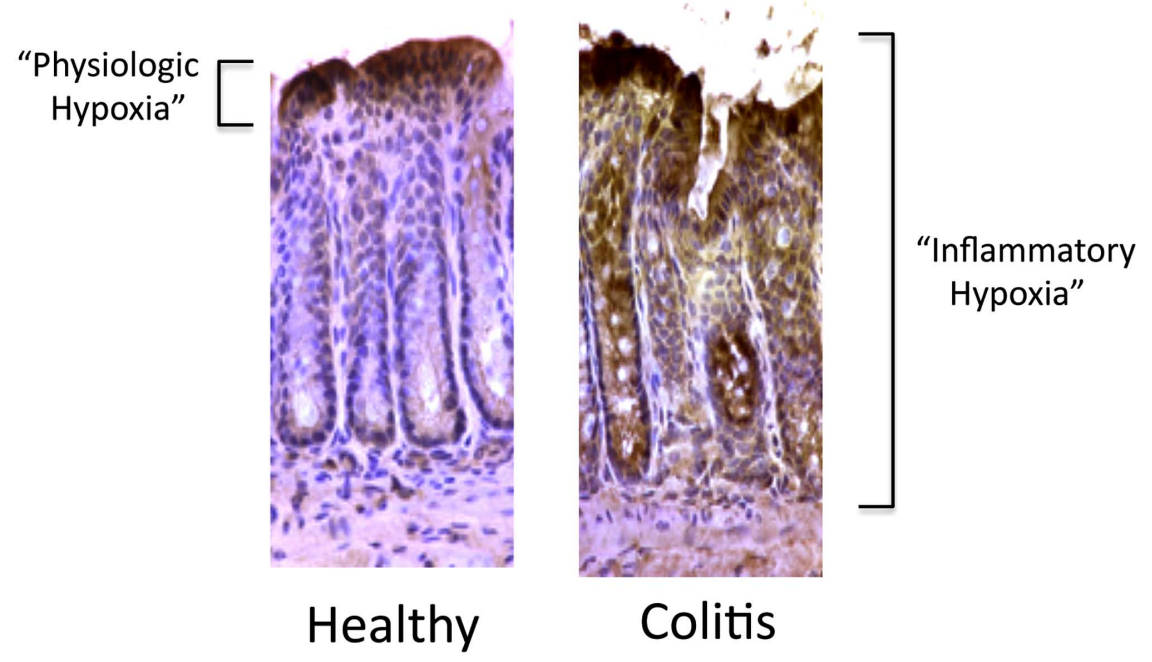

Fig. (2). Localization of hypoxic regions in murine colitis. The in vivo evidence for inflammation-associated hypoxia (so called "inflammatory hypoxia") are provided using nitroimidazole-based dye retention in mouse models of colitis. Nitroimidazole dyes passively absorbed into cells and reduced to highly reactive nitrogen intermediates. In the absence of adequate oxygen to regenerate the native compound, these intermediates react with cellular proteins to form adducts, which can be visualized using antibodies directed at these nitroimidazole adducts. In colonic tissue of mice with no inflammation (healthy) small amounts of nitroimidazole adduct is detected along the luminal aspect of the colon, depicted here as "physiological hypoxia". During episodes of inflammation, such as seen here in a mouse model of colitis, intense and deep tissue hypoxia is observed.

ate HIF target gene specificity via interactions with auxiliary transcription factors [14], but compelling evidence for this aspect remains inconclusive.

\section{INTRA-LUMINAL BARRIER AND HYPOXIA}

\section{Immunoglobulin A}

Secretion of immunoglobulin A $(\operatorname{Ig} \mathrm{A})$ into the intestinal lumen contributes to the integrity of the gut barrier. IgA has the capacity to neutralize toxins and to prevent microbial translocation from the lumen by inhibiting adhesion and invasion. A remarkable $46.5 \mathrm{mg} / \mathrm{kg}$ body weight of $\operatorname{IgA}$ is estimated to enter the digestive tract each day (3 grams for a $65 \mathrm{~kg}$ individual). Relatively small amounts are secreted in saliva and bile with over $90 \%$ being secreted across the intestinal epithelium, originating from plasma cells resident in the lamina propria, Peyer's patches, and isolated lymphoid follicles of the intestine $[19,20]$. In the intestine, polymeric IgA undergoes secretion by binding the polymeric Ig receptor expressed on the basolateral side of the enterocyte. This initiates transcytosis across the epithelium and into the lumen. The importance of intestinal IgA secretion is demonstrated in mice lacking the polymeric Ig receptor. These mice produce $\operatorname{IgA}$, but do not secrete it across mucosal surfaces. They exhibit evidence of compromised gut barrier function revealed by increase numbers of bacteria cultured from mesenteric lymph nodes and increased serum IgA and IgG specific for microbial antigens reflecting exposure to microbial components [21].

Few studies have investigated the influence of hypoxia on secretion of IgA in the gut. This is in part due to technical challenges caused by the lack of IgA transcytosis in commonly utilized intestinal epithelial derived cell lines [22]. One model overcomes this by utilizing Madin-Darby Canine Kidney (MDCK) cells transfected with the polymeric Ig re- ceptor [23]. The addition of $\operatorname{IgA}$ in this in vitro model attenuates bacterial translocation [22]. To examine the influence of oxygen tension in this system, MDCK monolayers were exposed to normoxia $\left(21 \% \mathrm{O}_{2}\right)$, hypoxia $\left(5 \% \mathrm{O}_{2}\right)$ or hyperoxia $\left(95 \% \mathrm{O}_{2}\right)$ for 90 minutes and transcytosis of $\operatorname{IgA}$ was measured from the basolateral to the apical chamber. No difference in transcytosis was detected between normoxia and hyperoxia. In contrast, hypoxia increased $\operatorname{IgA}$ transcytosis $(\mathrm{p}<0.001)$ at all time points $(1 \mathrm{~h}, 3 \mathrm{~h}$, and overnight) [24], despite the relatively short exposure. Notably, IgA contributes to epithelial barrier integrity in the context of hypoxia. This was demonstrated in intestinal epithelial (Caco2) monolayers exposed to 90 minutes of hypoxia with the addition of colostrum-derived polyclonal IgA prior to E. coli exposure. IgA prevented epithelial cell apoptosis that occurred with combined exposure to hypoxia and E. coli [25]. This is consistent with an in vivo description of the protective effect of orally supplemented $\operatorname{IgA}$, but not $\mathrm{IgG}$, on villous architecture and bacterial translocation in a neonatal rabbit model of milk formula induced injury [26]. The role of the HIF pathway in regulating $\operatorname{IgA}$ synthesis and transcytosis in the intestine remains to be determined. Furthermore, it is unclear if other models will reproduce the augmented IgA transcytosis following hypoxia exposure given that the protective role of HIF signaling in chronic inflammatory hypoxia [27, 28], has not always been in agreement with those of acute ischemia/ reperfusion [29].

\section{Mucins}

The intestinal mucus layer is a complex mixture of glycoproteins that buffer exposure of the host epithelium to the luminal contents. While permitting passage of nutrients to the epithelium, it simultaneously prevents access by microbes, toxins, and digestive enzymes. Additional barrier protective functions include lubrication of luminal contents, 
neutralization of free radicals, sequestration of antimicrobial peptides, providing "decoy" adhesion sites for pathogenic bacteria, and providing a substrate for commensal mucolytic microbes [30]. At least 10 of the 16 human mucin glycoproteins are found in the small intestine or colon. These include secreted gel forming (MUC2 and 6) and cell surface mucins (MUC3A/B , 4, 12, 13, 15, 17, and 20) [30]. In vivo measurement of the colonic mucus layer in rats determined the total thickness to average $642 \mu \mathrm{m}(101 \mu \mathrm{m}$ firmly adherent) in one study [31] and $830 \mu \mathrm{m}(116 \mu \mathrm{m}$ firmly adherent) in another [32]. Mean mucus thickness in the small intestine was less than the colon, measuring $170 \mu \mathrm{m}(16 \mu \mathrm{m}$ firmly adherent) in the duodenum, $123 \mu \mathrm{m}(15 \mu \mathrm{m}$ firmly adherent) in the jejunum, and $480 \mu \mathrm{m}(29 \mu \mathrm{m}$ firmly adherent) in the ileum [31]. This represents a formidable barrier many times thicker than the epithelium itself.

The influence of hypoxia on the expression of MUC genes in the intestines has only recently received attention. Using the T84 colon cancer cell line, MUC1 and MUC2 expression was not altered by exposure to 6 or 18 hours of hypoxia. However, MUC3 was shown to be induced by those conditions and to be regulated by HIF-1 $\alpha$ [33]. Maximal MUC3 protein expression occurred at 24 hours and, for unknown reasons, was reduced at 48 hours. One may speculate that increased MUC3 results in destabilization of HIF- $1 \alpha$ as has been shown to occur with MUC1, another cell surface mucin. This was demonstrated by Yin, et al. [34] in studies that used the HCT116 colon cancer cell line transfected to express MUC1 [35]. Using this model, they determined that stabilization of HIF-1 $\alpha$ following exposure to hypoxia was reduced in cells transfected to express MUC1, but not the vector-only control. Several observations could explain this. First, hypoxia can increase generation of reactive oxygen species by mitochondria [36]. This causes oxidation of $\mathrm{Fe}^{2+}$, a necessary cofactor for PHD mediated HIF-1 degradation, to $\mathrm{Fe}^{3+}$ [37]. Second, MUC1 has been shown to alleviate oxidative stress [38, 39]. Indeed, it was found that MUC1 decreases ROS and upregulates PHD3 leading to HIF- $1 \alpha$ degradation [34]. Because of this complex interaction, it is unclear what overall impact pharmacologic targeting of the HIF pathway would have on the mucin expression.

\section{Trefoil Peptides}

Mucin glycoproteins provide a framework that enables interaction with other secreted peptides. Trefoil factors, characterized by 38-39 amino acid "trefoil" domains, are one example of proteins that co-localize with mucins [40-42] and modulate mucin structure [41]. Trefoil domains assume a compact, clover-shaped, configuration enabled by three disulfide bonds [43] which render them resistant to proteolysis and low $\mathrm{pH}[44,45]$. Three mammalian trefoil factors, TFF1, TFF2, TFF3, have been identified and are variably expressed in mucus secreting epithelial tissues with prominent expression throughout the gastrointestinal tract. TFF1 is primarily expressed in the stomach [46] with expression in the intestine associated primarily with inflammation and mucosal damage [47, 48]. TFF2 is expressed in the gastric and Brunner's glands, while TFF3 is primarily expressed in the intestine and is also referred to as intestinal trefoil factor, or ITF $[43,44]$. Trefoil factor expression is markedly increased after mucosal injury and has been shown to have anti- apoptotic, pro-angiogenic, pro-migratory, and immunomodulatory influences at mucosal surfaces that together enable rapid restitution of the barrier following insult (reviewed in [43]).

TFF3-null mice are more susceptible to dextran sodium sulphate induced colitis [49] while selective over-expression of TFF1 [50] and TFF3 [51] in the jejunum offers protection from indomethacin induced ulceration. Little is known about trefoil factor expression in human intestinal disease. No difference in TFF3 staining was observed in biopsies from ulcerative colitis patients vs. controls [52]. One study of duodenal biopsies from patients with celiac disease identified lower levels of TFF3 transcript and protein in untreated patients. Interestingly adherents to a gluten-free diet had levels comparable to healthy controls [53]. In contrast, TFF1 transcript was increased in 16 untreated pediatric subjects with celiac disease compared to 9 adherents to a gluten-free diet. HIF- $1 \alpha$ transcript and protein was also measured and found to be increased in the untreated group [54].

The HIF pathway is one link between mucosal injury and trefoil factor expression. Furuta, et al. found transcription of TFF3 induced in Caco-2 and T84 intestinal epithelial cells by exposure to hypoxia. They identified the HIF binding site on the cloned TFF3 promoter, demonstrated that over expression and knockdown of HIF-1 $\alpha$ correspondingly augmented and decreased TFF3 protein levels, and observed a dose-dependent decreased flux of FITC-labeled dextran across monolayers with the addition of recombinant TFF3. Finally, the in vivo contribution of TFF3 to barrier function was established using TFF3 null mice. Intestinal permeability to FITC-labeled dextran was higher under conditions of both normoxia and hypoxia. The significance of HIF- $1 \alpha$ regulation of TFF3 was underscored by the death of 4 of 6 TFF3-null mice exposed to 18 hours of hypoxia (8\% O2), while all 6 wild-type mice survived the same exposure [55]. Interestingly, co-precipitation of MUC3 with anti-TTF3 antibodies [33] demonstrated interaction between these two HIF- $1 \alpha$ regulated proteins and supporting the importance of HIF signaling on maintenance of the intraluminal barrier.

\section{TIGHT JUNCTION REGULATION IN HYPOXIA}

Through interactions with the intracellular cytoskeleton, tight junctions form the backbone to the structural integrity of the barrier and constitute the physical basis for the permeability barrier to solutes and ions (Fig. 1). Contributing to the polarized phenotype of epithelia, they furthermore prevent lipid diffusion between apical and basolateral membrane domains, the so called "fence function" of tight junctions [3]. The tight junction is composed of both transmembrane and peripheral membrane proteins, which are linked to the actinbased cytoskeleton [3] where both complex assembly and transcriptional control of its components is tightly regulated by a variety of physiological and pathophysiological stimuli. Ischemia dramatically affects tight junction integrity resulting in loss of transepithelial electrical resistance which has been observed both in ATP depletion models [56] and in vitro hypoxia models $[2,57,58]$. Some of the permeability changes are attributed to alterations in distribution of occludin, zonula occludens-1 (ZO-1), ZO-2 and cingulin [56, 
59]. Furthermore, vascular endothelial growth factor (VEGF) is a generally accepted to be a hypoxia compensatory mechanism initiating increased angiogenesis in regions of reduced oxygen supply. The underlying mechanism involves the loss of occludin organization apparently through the activation of extracellular signal regulated kinases (ERK1/2) [60] and through NO dependent mechanisms [61]. Furthermore, tight junction integrity is influenced by perturbations of the interaction with the actin-based cytoskeleton [62, 63] and by the degradation of such membrane-cytoskeletal proteins as ankyrin and fodrin both in ATP depletion models [64] and in endothelial hypoxia [65].

\section{Cyclic Nucleotides in Barrier Regulation}

Prompted by the observation that hypoxic cells have a lessened capacity to generate cyclic nucleotides [66], a number of studies have addressed the role of cyclic adenosine monophosphate (cAMP) on tight junction permeability. In general, changes in cAMP do not significantly impact tight junction permeability of the intact epithelium [67]. Reestablishment of disrupted epithelia, termed restitution, is however, significantly dependent on adequate generation of cAMP. For example, it was shown that post-hypoxic epithelia fail to normally re-develop barrier following either physiologic disruption (e.g. neutrophil migration, see Fig. 3) or modeled disruption (e.g. calcium depletion), and that such defects were at least in part attributed to diminished cAMP generation [58]. At present, it is unclear why cAMP may be so critical to restitution. Recent studies have suggested that increases intracellular cAMP levels may promote barrier function through different mechanisms including increased expression of tight junctional proteins $\mathrm{ZO}-1$ and occludin [68] and an increase of mean number of tight-junction strands [69]. Furthermore increases in cAMP levels are accompanied by an increase in polymerized actin and increased phosphorylation of intermediate filaments, suggesting that cAMP-mediated changes in permeability may be due to al- terations in the cellular cytoskeleton [70]. Because of the actin-binding and cross linking functions of vasodilatorstimulated phosphoprotein (VASP), its protein kinase A (PKA) mediated phosphorylation may be crucial in this pathway. This work revealed that VASP localizes with ZO-1 at the tight junction and appears as phospho-VASP at the junction following $\mathrm{Ca}^{2+}$ switch [71]. Apart from the increasing insights that link cAMP signal transduction to tight junctional organization, cAMP affects permeability through modulation of the junctional expression of adherens junction proteins VE-Cadherin and PECAM-1 [68] and through cAMP-dependent phosphorylation of VE-cadherin [72]. Finally, compelling evidence indicates that oxidative stress, commonly associated with hypoxia-reoxygenation, directly influences the structure of the endothelial [60] and epithelial tight junction $[73,74]$.

\section{Nucleotide Metabolism in Barrier Regulation}

As depicted in Fig. (3), circulating or locally released nucleotides are rapidly metabolized by ecto-enzymes localized on the cell surface. Ecto-5'-nucleotidase (CD73) is a glycosyl phosphatidylinositol (GPI)-linked, membranebound glycoprotein which hydrolyzes extracellular nucleoside monophosphates into bioactive nucleoside intermediates [75]. Surface-bound CD73 metabolizes adenosine 5'monophosphate (AMP) to adenosine, which when released can activate one of four types of G-protein coupled, seven transmembrane spanning adenosine receptors (AdoR) or can be internalized through dipyridamole-sensitive carriers [76]. Adenosine receptors are expressed on a wide variety of cells, and many cell types have been shown to express more than one isoform of the receptor. Likewise, activation of surface AdoR has been shown to regulate diverse physiologic endpoints. In the recent years, our understanding of nucleotide metabolic pathways has benefited from the development of genetically manipulated animals, particularly mice deficient in $C d 73$ or a second nucleotide metabolizing enzyme, $C d 39$

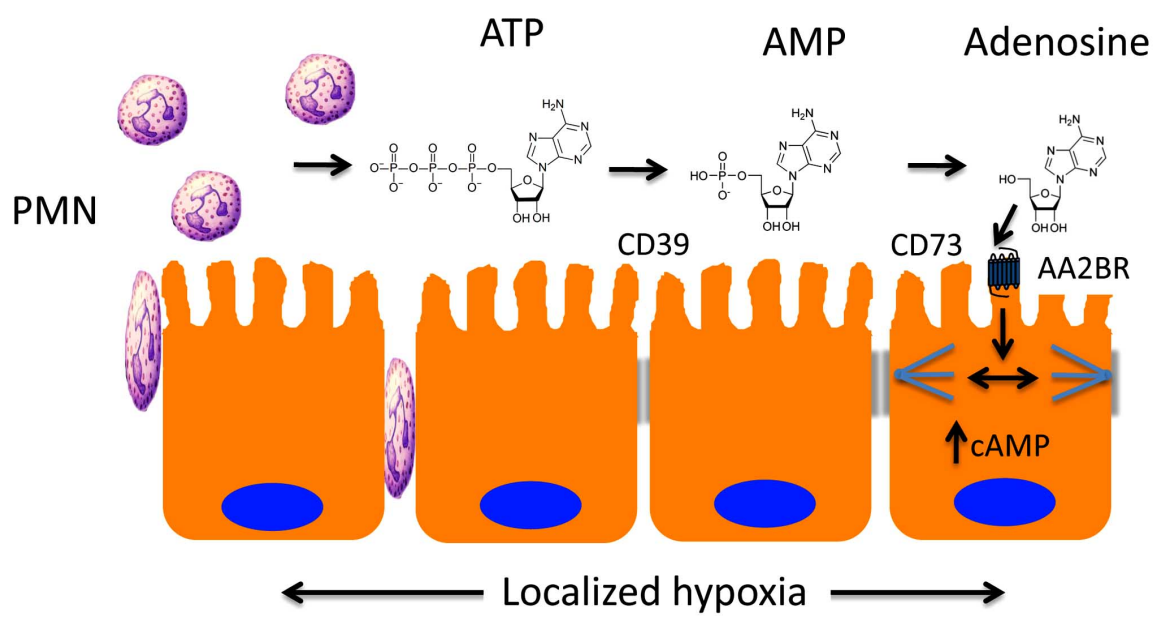

Fig. (3). Dynamic regulation of barrier function during inflammatory hypoxia. During episodes of mucosal inflammation, inflammatory hypoxia coordinates changes that lead to the dynamic regulation of epithelial tight junctions. As depicted here, these changes can involve nucleotide-mediated biochemical crosstalk between migration neutrophils (PMN) and epithelial cells. Such extracellular metabolism of involves the two-step phosphohydrolysis of ATP by surface expressed CD39 (or CD39-like enzymes) and CD73 to liberate adenosine. Extracellular adenosine generated in this manner activates surface receptors that signal to the cytoskeleton and dynamically regulate tight junction permeability. Hypoxia coordinates this biochemical crosstalk through transcriptional regulation of CD39, CD73 and the A2B adenosine receptor (AA2BR). 
(ecto-apyrase), that catalyzes the phosphohydrolysis of ATP and ADP to AMP.

A number of studies have implicated CD39 and CD73 in the control of tissue barrier function, particularly in during hypoxia. Successful transmigration of leukocytes, especially polymorphonuclear (PMN, neutrophil) leukocytes across endothelia and epithelia is accomplished by temporary selfdeformation with localized widening of the inter-junctional spaces [77], a process with the potential to disturb endothelial and epithelial barrier function. Original studies by Lennon et al. revealed that the prominent signaling pathway for closing inter-endothelial gaps during neutrophil transmigration involved adenosine-induced "restitution" of barrier [78]. Until recently, only limited information existed regarding the biochemical events which regulate cellular barriers in the setting of either neutrophil activation or transmigration [79, 80]. Lennon et al. showed it that inhibition of CD73 using either APCP or anti-CD73 monoclonal antibody 1E9 inhibited the resealing of endothelial and epithelial barriers by as much as $85 \%$ [78], suggesting the necessity for extracellular nucleotide metabolism in this pathway. Subsequent studies revealed that adenosine produced from neutrophil-derived AMP was responsible for enhanced barrier function via activation of the adenosine A2B receptor coupling to cytoskeletal links[81]. More recently, it was shown that in addition to (or rather than) releasing AMP, neutrophils actively release ATP following receptor-mediated stimulation[82]. Such ATP is hydrolyzed to adenosine at the endothelial cell surface through the coordinated actions of CD39 and CD73 It is not clear exactly how neutrophils and / or endothelial cells release ATP, although several mechanisms have been proposed, including direct transport through ATP-binding cassette $(\mathrm{ABC})$ proteins, transport through connexin hemichannels, as well as vesicular release [83].

CD73 lies central to the regulation of tissue barriers during episodes of hypoxia (see Fig. 3). Studies have revealed that CD73 is a strongly HIF-regulated gene and is critically important for the generation of extracellular adenosine in hypoxia [84]. Studies in mouse models of increased intestinal permeability revealed that oral delivery the CD73 inhibitor APCP promotes movement of inert tracers, such as FITClabelled dextran, across the intestinal epithelium [84]. Likewise, to investigate hypoxia-induced changes in tissue permeability in $C d 73^{-/}$mice [85], we have used Evan's blue dye, which binds tightly to plasma albumin [86]. Quantification of formamide-extractable Evan's blue from individual tissues can then be interpreted as a function of vascular leak [87]. In general, hypoxia increases vascular permeability two- to four-fold over normoxic conditions, depending on the tissue [88]. Pharmacologic interventions have suggested that CD73 is protective under such circumstances, and most studies have implicated a protective role for adenosine A2A (AA2AR) and A2B (AA2BR) receptors in maintaining barrier function $[89,90]$. These studies have defined CD39 and CD73 as the pacemakers for the fine-tuning of epithelial and endothelial permeability. Such innate protective pathways share the common strategy of increasing extracellular adenosine concentrations and promoting adenosine signaling from the cell surface through the cytoskeleton and ultimately to dynamic regulation of tight junctions.

\section{TARGETING HYPOXIA PATHWAYS}

\section{HIF and Barrier Function}

A number of studies have shown that HIF triggers the expression of genes that enable intestinal epithelial cells to function as an effective barrier $[2,84,88,91]$. Originally shown by microarray analysis of hypoxic intestinal epithelial cells [84], these studies have been validated in animal models of intestinal inflammation [11,92-96] and in inflamed human intestinal tissues [97-99]. The functional proteins encoded by hypoxia-induced, HIF-dependent mRNAs localize primarily to the most luminal aspect of polarized epithelia. Molecular studies of these hypoxia-elicited pathway(s) have shown a dependence on HIF-mediated transcriptional responses. Notably, epithelial barrier protective pathways driven by HIF tend not to be the classical regulators of barrier function, such as the tight junction proteins occludin or claudins. Rather, the HIF-regulated pathways are more to do with overall tissue integrity, ranging from increased mucin production [100], including molecules that modify mucins, such as, intestinal trefoil factor [2], to xenobiotic clearance by P-glycoprotein [91], to nucleotide metabolism (by ecto-5'nucleotidase and CD73) $[84,88]$ and nucleotide signaling through the adenosine A2B receptor [88].

As an extension of the original studies identifying HIF induction within the intestinal mucosa, Karhausen, et al. generated mice expressing either mutant Hifla (causing constitutive repression of Hifla) or mutant von Hippel-Lindau (causing constitutive overexpression of HIF) targeted to the intestinal epithelial cells [11]. Loss of epithelial HIF-1 $\alpha$ resulted in a more severe colitic phenotype than wild-type animals, with increased weight loss, decreased colon length and increased intestinal permeability, whereas constitutively active intestinal epithelial HIF was protective for each of these parameters. These findings were somewhat modeldependent, since epithelial HIF-based signaling has also been shown to promote inflammation in another study [96]. However, the findings confirmed that intestinal epithelial cells can adapt to hypoxia and that HIF may contribute such adaptation.

\section{HIF Prolyl-hydroxylases}

In the past 10 years, the molecular mechanisms of HIF stabilization have been clarified. Four HIF-hydroxylases termed PHD1-3 and Factor Inhibiting HIF-1 (FIH) have been demonstrated to be important in the hypoxic regulation of the HIF pathway [101]. Each of these hydroxylases are encoded by different genes and their gene product enzymes demonstrate tissue specific expression patterns [101]. All three PHD's and FIH are found in the intestinal epithelium [92, 95, 102]. Significantly, different phenotypes in mice genetically lacking individual isoforms of the hydroxylases exist. For instance, $\mathrm{PHD}^{-/}$mice demonstrate a reprogrammed basal metabolic profile in normal tissue which decreases exercise performance but these animals are protected against acute liver and muscle ischemia [103, 104]. PHD2 homozygous knockout is embryonic lethal due to developmental angiogenesis dysfunction [105, 106]. PHD2 heterozygous knockout animals demonstrate enhanced tumor angiogenesis but decreased metastasis [105]. PHD3 homozygous knockout mice demonstrate reduced neuronal apop- 


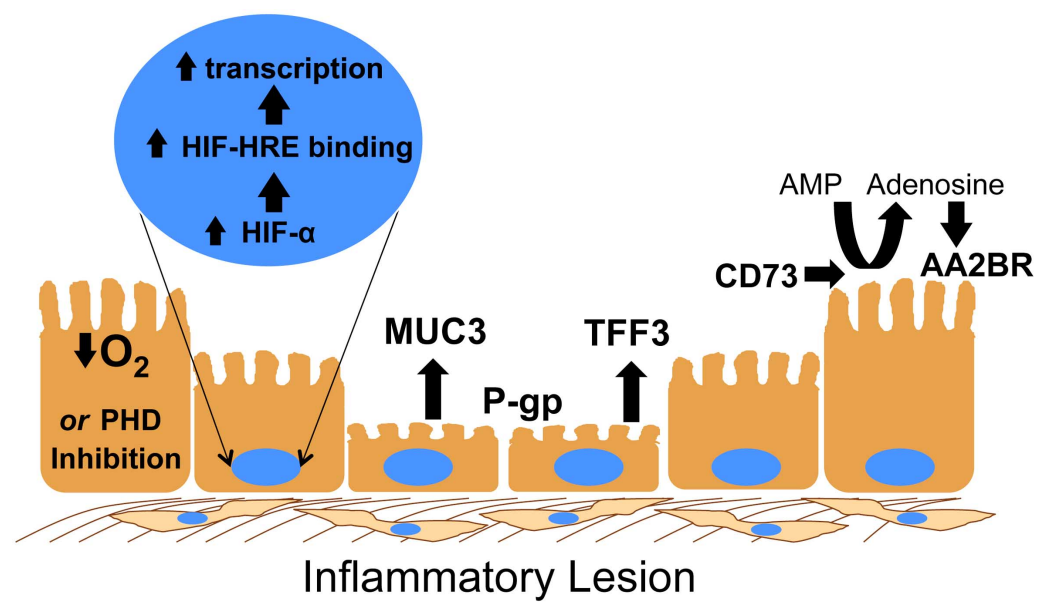

Fig. (4). Targeting epithelial HIF- $\alpha$ to augment the response to ypoxia. Low oxygen tension or pharmacologic inhibition of HIF- $\alpha$ degrading (PHD) enzymes upregulates expression of genes protective for epithelial barrier function including mucins (e.g. Muc-3), intestinal trefoil factor (TTF3) and P-glycoprotein (P-gp, Mdr-1 gene product). Increased expression of CD73 and the AA2B receptor enhance protective adenosine signaling.

tosis, abnormal sympathoadrenal development and reduced blood pressure [107]. These diverse phenotypes strongly suggest distinct isoform-specific functions in vivo.

The discovery of HIF-selective PHD's as central regulators of HIF expression has now provided the basis for potential development of PHD-based molecular tools and therapies [108, 109]. Pharmacological inactivation of the PHDs by 2-oxoglutarate analogues is sufficient to stabilize HIF$1 \alpha$ [108], but this action is nonspecific with respect to individual PHD isoforms. In vitro studies suggest some significant differences in substrate specificity. For example, PHD3 does not hydroxylate proline 564 on $\mathrm{HIF}-\alpha$, and comparison of enzyme activity in vitro showed that the oxygendependent degradation sequence is hydroxylated most efficiently by PHD2 $[110,111]$. These observations have generated significant interest in identifying enzyme-modifying therapeutics. Indeed, a number of PHD inhibitors have been described, including direct inhibitors of the prolylhydroxylases [112], analogs of naturally occurring cyclic hydroxamates [113], as well as antagonists of alpha-ketoglutarate [108]. As such, we have hypothesized that pharmacologic activation of HIF would afford protection in murine colitic disease. For these purposes, we and others have used prolyl hydroxylase inhibitors which stabilize HIF-1 $\alpha$ and subsequently drive the expression of downstream HIF target genes. These results show that the PHD inhibition provides an overall beneficial influence on clinical symptoms (weight loss, colon length, tissue TNF $\alpha$ / IFN $\gamma$ ) in murine TNBS or DSS colitis models, most likely due to their barrier protective function and wound healing during severe tissue hypoxia at the site of inflammation $[92,95]$. These findings emphasize the role of epithelial HIF-1 $\alpha$ during inflammatory diseases in the colon and may provide the basis for a therapeutic use of PHD inhibitors in inflammatory mucosal disease.

Importantly, it is recently appreciated that the oxygendependent regulatory role of hydroxylases may not be restricted to the HIF pathway. Indeed, some studies have indicated that the NF- $\mathrm{KB}$ pathways may also be regulated by hydroxylases. Hypoxia activates NF- $\mathrm{KB}$ and this appears, at least in part, to be mediated through altered hydroxylation of critical components of this pathway [102]. Interestingly, like intestinal epithelial conditional HIF-1 $\alpha$-null mice, conditional deletion of the $\mathrm{NF}-\kappa \mathrm{B}$ in intestinal epithelia leads to increased susceptibility to colitis indicating a protective role for epithelial $\mathrm{NF}-\kappa \mathrm{B}$ in colitis, likely through the expression of anti-apoptotic genes in the intestinal epithelium resulting in enhanced epithelial barrier function [114]. Thus, a part of the protective influence of hydroxylase inhibition in models of colitis may be through the promotion of intestinal epithelial NF- $\kappa \mathrm{B}$ activity.

\section{CONCLUSION}

The gastrointestinal mucosa provides a unique setting to study tissue oxygenation and barrier regulation. Studies showing relatively low baseline $\mathrm{pO}_{2}$ coupled with high energy demand on a backdrop of inflammatory activity have revealed this mucosal surface as an interesting target for HIF-based barrier augmentation. Results from in vitro and in vivo models of IBD have demonstrated an overall the beneficial impact of HIF stabilization via HIF hydroxylase inhibition. A common mechanistic thread throughout these studies includes the regulation of mucosal barrier function. Additional studies will be necessary to better define these concepts, including specific gene targets, mechanisms of anti-inflammation and the potential for tissue-specific HIF PHD expression (Fig. 4). If successful, this strategy may provide an approach to augment barrier function by pharmacological means.

\section{CONFLICT OF INTEREST}

None declared.

\section{ACKNOWLEDGEMENT}

This work was supported by National Institutes of Health Grants DK50189 and HL60569 and by a grant from the Crohn's and Colitis Foundation of America.

\section{REFERENCES}

[1] Schulzke JD, Ploeger S, Amasheh M, et al. Epithelial tight junctions in intestinal inflammation. Ann NY Acad Sci 2009; 1165 : 294-300. 
[2] Furuta GT, Turner JR, Taylor CT, et al. Hypoxia-inducible factor 1-dependent induction of intestinal trefoil factor protects barrier function during hypoxia. J Ex Med 2001; 193: 1027-34.

[3] Ivanov AI, Parkos CA, Nusrat A. Cytoskeletal regulation of epithelial barrier function during inflammation. Am J Pathol 2010; 177: 512-24.

[4] Turner JR. Intestinal mucosal barrier function in health and disease. Nat Rev Immunol 2009; 9: 799-809.

[5] Kominsky DJ, Campbell EL, Colgan SP. Metabolic shifts in immunity and inflammation. J Immunol 2010; 184: 4062-8.

[6] Borregaard N, Herlin T. Energy metabolism of human neutrophils during phagocytosis. J Clin Invest 1982; 70: 550-7.

[7] El-Benna J, Dang PM, Gougerot-Pocidalo MA. Priming of the neutrophil NADPH oxidase activation: role of p47phox phosphorylation and NOX2 mobilization to the plasma membrane. Semin Immunopathol 2008; 30: 279-89.

[8] Gabig TG, Bearman SI, Babior BM. Effects of oxygen tension and $\mathrm{pH}$ on the respiratory burst of human neutrophils. Blood 1979; 53: 1133-9.

[9] Taylor CT, Colgan SP. Hypoxia and gastrointestinal disease. J Mol Med 2008; 85: 1295-300.

[10] Evans SM, Hahn S, Pook DR, et al. Detection of hypoxia in human squamous cell carcinoma by EF5 binding. Cancer Res 2000; 60: 2018-24.

[11] Karhausen JO, Furuta GT, Tomaszewski JE, et al. Epithelial hypoxia-inducible factor- 1 is protective in murine experimental colitis. J Clin Invest 2004; 114: 1098-106.

[12] Semenza GL. Regulation of oxygen homeostasis by hypoxiainducible factor 1. Physiology 2009; 24: 97-106.

[13] Schofield CJ, Ratcliffe PJ. Oxygen sensing by HIF hydroxylases. Nat Rev Mol Cell Biol 2004; 5: 343-54.

[14] Ratcliffe PJ. HIF-1 and HIF-2: working alone or together in hypoxia? J Clin Invest 2007; 117: 862-5.

[15] Mastrogiannaki M, Matak P, Keith B, et al. HIF-2alpha, but not HIF-1alpha, promotes iron absorption in mice. J Clin Invest 2009; 119: 1159-66.

[16] Hu CJ, Wang LY, Chodosh LA, et al. Differential roles of hypoxiainducible factor 1alpha (HIF-1alpha) and HIF-2alpha in hypoxic gene regulation. Mol Cell Biol 2003; 23: 9361-74.

[17] Mastrogiannaki M, Matak P, Keith B, et al. HIF-2alpha, but not HIF-1alpha, promotes iron absorption in mice. J Clin Invest 2009; 119: 1159-66.

[18] Rankin EB, Biju MP, Liu Q, et al. Hypoxia-inducible factor-2 (HIF-2) regulates hepatic erythropoietin in vivo. J Clin Invest 2007; 117: 1068-77.

[19] Conley ME, Delacroix DL. Intravascular and mucosal immunoglobulin A: two separate but related systems of immune defense? Ann Intern Med 1987; 106: 892-9.

[20] Suzuki K, Fagarasan S. Diverse regulatory pathways for IgA synthesis in the gut. Mucosal Immunol 2009; 2: 468-71.

[21] Sait LC, Galic M, Price JD, et al. Secretory antibodies reduce systemic antibody responses against the gastrointestinal commensal flora. Int Immunol 2007; 19: 257-65.

[22] Diebel LN, Liberati DM, Brown WJ, et al. Secretory immunoglobulin A blocks hypoxia-augmented bacterial passage across Madin-Darby canine kidney cell monolayers. J Trauma 1997; 43: 759-63.

[23] Mostov KE, Deitcher DL. Polymeric immunoglobulin receptor expressed in MDCK cells transcytoses IgA. Cell 1986; 46: 613-21.

[24] Baylor AE, Diebel LN, Liberati DM, et al. The effects of varying oxygen conditions and immunoglobulin $\mathrm{A}$ on barrier defense to bacterial invasion. Am Surg 2003; 69: 231-7.

[25] Diebel LN, Liberati DM, Dulchavsky SA, et al. Enterocyte apoptosis and barrier function are modulated by SIgA after exposure to bacteria and hypoxia/reoxygenation. Surgery 2003; 134: 574-80.

[26] Dickinson EC, Gorga JC, Garrett M, et al. Immunoglobulin A supplementation abrogates bacterial translocation and preserves the architecture of the intestinal epithelium. Surgery 1998; 124: 284-90.

[27] Cummins EP, Seeballuck F, Keely SJ, et al. The hydroxylase inhibitor dimethyloxalylglycine is protective in a murine model of colitis. Gastroenterology 2008; 134: 156-65.

[28] Karhausen J, Furuta GT, Tomaszewski JE, et al. Epithelial hypoxiainducible factor- 1 is protective in murine experimental colitis. $\mathbf{J}$ Clin Invest 2004; 114: 1098-106.

[29] Feinman R, Deitch EA, Watkins AC, et al. HIF-1 mediates pathogenic inflammatory responses to intestinal ischemia-reperfusion injury. Am J Physiol Gastrointest Liver Physiol 2010; 299: G833-43.
[30] Linden SK, Sutton P, Karlsson NG, et al. Mucins in the mucosal barrier to infection. Mucosal Immunol 2008; 1: 183-97.

[31] Atuma C, Strugala V, Allen A, et al. The adherent gastrointestinal mucus gel layer: thickness and physical state in vivo. Am J Physiol Gastrointest Liver Physiol 2001; 280: G922-9.

[32] Strugala V, Allen A, Dettmar PW, et al. Colonic mucin: methods of measuring mucus thickness. Proc Nutr Soc 2003; 62: 237-43.

[33] Louis NA, Hamilton KE, Canny G, et al. Selective induction of mucin-3 by hypoxia in intestinal epithelia. J Cell Biochem 2006; 99: 1616-27.

[34] Yin L, Kharbanda S, Kufe D. Mucin 1 oncoprotein blocks hypoxiainducible factor 1alpha activation in a survival response to hypoxia. J Biol Chem 2007; 282: 257-66.

[35] Ren J, Agata N, Chen D, et al. Human MUC1 carcinomaassociated protein confers resistance to genotoxic anticancer agents. Cancer Cell 2004; 5: 163-75.

[36] Chandel NS, McClintock DS, Feliciano CE, et al. Reactive oxygen species generated at mitochondrial complex III stabilize hypoxiainducible factor-1alpha during hypoxia: a mechanism of $\mathrm{O} 2$ sensing. J Biol Chem 2000; 275: 25130-8.

[37] Gerald D, Berra E, Frapart YM, et al. JunD reduces tumor angiogenesis by protecting cells from oxidative stress. Cell 2004; 118: 781-94.

[38] Yin L, Huang L, Kufe D. MUC1 oncoprotein activates the FOXO3a transcription factor in a survival response to oxidative stress. J Biol Chem 2004; 279: 45721-7.

[39] Yin L, Li Y, Ren J, et al. Human MUC1 carcinoma antigen regulates intracellular oxidant levels and the apoptotic response to oxidative stress. J Biol Chem 2003; 278: 35458-64.

[40] Longman RJ, Douthwaite J, Sylvester PA, et al. Coordinated localisation of mucins and trefoil peptides in the ulcer associated cell lineage and the gastrointestinal mucosa. Gut 2000; 47: 792-800.

[41] Thim L, Madsen F, Poulsen SS. Effect of trefoil factors on the viscoelastic properties of mucus gels. Eur J Clin Invest 2002; 32: 519-27.

[42] Williams GR, Wright NA. Trefoil factor family domain peptides. Virchows Arch 1997; 431: 299-304.

[43] Thim L. Trefoil peptides: from structure to function. Cell Mol Life Sci 1997; 53: 888-903.

[44] Kjellev S. The trefoil factor family - small peptides with multiple functionalities. Cell Mol Life Sci 2009; 66: 1350-69.

[45] Kjellev S, Vestergaard EM, Nexo E, et al. Pharmacokinetics of trefoil peptides and their stability in gastrointestinal contents. Peptides 2007; 28: 1197-206.

[46] Tomasetto C, Rio MC. Pleiotropic effects of Trefoil Factor 1 deficiency. Cell Mol Life Sci 2005; 62: 2916-20.

[47] Rio MC, Chenard MP, Wolf C, et al. Induction of pS2 and hSP genes as markers of mucosal ulceration of the digestive tract. Gastroenterology 1991; 100: 375-9.

[48] Vannay A, Sziksz E, Prokai A, et al. Increased expression of hypoxia-inducible factor 1alpha in coeliac disease. Pediatr Res 2010; 68: $118-22$.

[49] Beck PL, Ihara E, Hirota SA, et al. Exploring the interplay of barrier function and leukocyte recruitment in intestinal inflammation by targeting fucosyltransferase VII and trefoil factor 3. Am J Physiol Gastrointest Liver Physiol 2010; 299: G43-53.

[50] Playford RJ, Marchbank T, Goodlad RA, et al. Transgenic mice that overexpress the human trefoil peptide $\mathrm{pS} 2$ have an increased resistance to intestinal damage. Proc Natl Acad Sci USA 1996; 93 : 2137-42.

[51] Marchbank T, Cox HM, Goodlad RA, et al. Effect of ectopic expression of rat trefoil factor family 3 (intestinal trefoil factor) in the jejunum of transgenic mice. J Biol Chem 2001; 276: 24088-96.

[52] Hamer HM, Jonkers DM, Renes IB, et al. Butyrate enemas do not affect human colonic MUC2 and TFF3 expression. Eur J Gastroenterol Hepatol 2010; 22: 1134-40.

[53] Ciacci C, Di Vizio D, Seth R, et al. Selective reduction of intestinal trefoil factor in untreated coeliac disease. Clin Exp Immunol 2002; 130: 526-31.

[54] Vannay A, Sziksz E, Prokai A, et al. Increased expression of hypoxia-inducible factor 1alpha in coeliac disease. Pediatr Res 2010; 68: 118-22.

[55] Furuta GT, Turner JR, Taylor CT, et al. Hypoxia-inducible factor 1-dependent induction of intestinal trefoil factor protects barrier function during hypoxia. J Exp Med 2001; 193: 1027-34. 
[56] Tsukamoto T, Nigam SK. Role of tyrosine phosphorylation in the reassembly of occludin and other tight junction proteins. Am J Physiol Renal Physiol 1999; 276: F737-F50.

[57] Taylor CT, Dzus AL, Colgan SP. Autocrine regulation of intestinal epithelial permeability by hypoxia: Role for polarized release of tumor necrosis factor alpha. Gastroenterology 1998; 114: 657-68.

[58] Friedman GB, Taylor CT, Parkos CA, et al. Epithelial permeability induced by neutrophil transmigration is potentiated by hypoxia: role of intracellular cAMP. J Cell Physiol 1998; 176: 76-84.

[59] Tsukamoto T, Nigam SK. Tight junction proteins form large complexes and associate with the cytoskeleton in an ATP depletion model for reversible junction assembly. J Biol Chem 1997; 272: 16133-9.

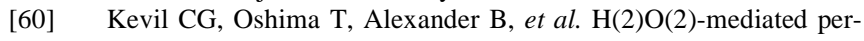
meability: role of MAPK and occludin. Am J Physiol Cell Physiol 2000; 279: C21-30.

[61] Wu HM, Huang QB, Yuan Y, et al. VEGF induces NO-dependent hyperpermeability in coronary venules. Am J Physiol Heart Circ Physiol 1996; 271: H2735-9.

[62] Molitoris BA, Leiser J, Wagner MC. Role of the actin cytoskeleton in ischemia-induced cell injury and repair. Pediatr Nephrol 1997; 11: 761-7.

[63] Bacallao RA, Garginkel A, Monke S, et al. ATP depletion: a novel method to study junctional properties in epithelial tissues. I. Rearrangement of the actin cytoskeleton. J Cell Sci 1994; 107: 3301-13.

[64] Molitoris BA, Dahl R, Hosford M. Cellular ATP depletion induces disruption of the spectrin cytoskeletal network. Am J Physiol 1996; 271: f790-8.

[65] Zharikov SI, Block ER. Association of L-arginine transporters with fodrin: implications for hypoxic ihibition of arginine uptake. Am J Physiol 2000; 278: L111-7.

[66] Hochachka PW, Buck LT, Doll CJ, et al. Unifying theory of hypoxia tolerance: molecular/metabolic defense and rescue mechanisms for surviving oxygen lack. Proc Natl Acad Sci USA 1996; 93: 9493-8.

[67] Shapiro M, Matthews J, Hecht G, et al. Stablization of F-actin prevents cAMP-elicited $\mathrm{Cl}$ - secretion in T84 cells. J Clin Invest 1991; 87: 1903-9.

[68] Dye JF, Leach L, Clark P, et al. Cyclic AMP and acidic fibroblast growth factor have opposing effects on tight and adherens junctions in microvascular endothelial cells in vitro. Microvasc Res 2001; 62: 94-113.

[69] Adamson RH, Liu B, Fry GN, et al. Microvascular permeability and number of tight junctions are modulated by cAMP. Am J Physiol 1998; 274: H1885-94.

[70] Stelzner TJ, Weil JV, O'Brien RF. Role of cyclic adenosine monophosphate in the induction of endothelial barrier properties. J Cell Physiol 1989; 139: 157-66.

[71] Lawrence DW, Comerford KM, Colgan SP. Role of VASP in reestablishment of epithelial tight junction assembly after $\mathrm{Ca} 2+$ switch. Am J Physiol Cell Physiol 2002; 282: C1235-45.

[72] Dejana E. Endothelial adherens junctions: implications in the control of vascular permeability and angiogenesis. J Clin Invest 1996; 98: 1949-53.

[73] Cuzzocrea S, Mazzon E, De Sarro A, et al. Role of free radicals and poly(ADP-ribose) synthetase in intestinal tight junction permeability. Mol Med 2000; 6: 766-78.

[74] Meyer TN, Schwesinger C, Ye J, et al. Reassembly of the tight junction after oxidative stress depends on tyrosine kinase activity. $\mathrm{J}$ Biol Chem 2001; 276: 22048-55.

[75] Zimmermann H, Braun N. Ecto-nucleotidases--molecular structures, catalytic properties, and functional roles in the nervous system. Prog Brain Res 1999; 120: 371-85.

[76] Linden J. Molecular approach to adenosine receptors: receptormediated mechanisms of tissue protection. Annu Rev Pharmacol Toxicol 2001; 41: 775-87.

[77] Chin AC, Parkos CA. Pathobiology of neutrophil transepithelial migration: implications in mediating epithelial injury. Annu Rev Pathol 2007; 2: 111-43.

[78] Lennon PF, Taylor CT, Stahl GL, et al. Neutrophil-derived 5'adenosine monophosphate promotes endothelial barrier function via CD73-mediated conversion to adenosine and endothelial $\mathrm{A}_{2 \mathrm{~B}}$ receptor activation. J Exp Med 1998; 188: 1433-43.

[79] Dejana E, Spagnuolo R, Bazzoni G. Interendothelial junctions and their role in the control of angiogenesis, vascular permeability and leukocyte transmigration. Thromb Haemost 2001; 86: 308-15.
[80] Ley K. Plugging the leaks. Nat Med 2001; 7: 1105-6.

[81] Comerford KM, Lawrence DW, Synnestvedt K, et al. Role of vasodilator-stimulated phosphoprotein in PKA-induced changes in endothelial junctional permeability. FASEB J 2002; 16: 583-5.

[82] Eltzschig HK, Thompson LF, Karhausen J, et al. Endogenous adenosine produced during hypoxia attenuates neutrophil accumulation: Coordination by extracellular nucleotide metabolism. Blood 2004; 104: 3986-92.

[83] Novak I. ATP as a signaling molecule: the exocrine focus. News Physiol Sci 2003; 18: 12-7.

[84] Synnestvedt K, Furuta GT, Comerford KM, et al. Ecto-5'nucleotidase (CD73) regulation by hypoxia-inducible factor-1 (HIF-1) mediates permeability changes in intestinal epithelia. J Clin Invest 2002; 110: 993-1002.

[85] Thompson LF, Eltzschig HK, Ibla JC, et al. Crucial role for ecto-5'nucleotidase (CD73) in vascular leak during hypoxia. J Exp Med 2004; 200: 1395-405.

[86] McDonald DM, Thurston G, Baluk P. Endothelial gaps as sites for plasma leakage in inflammation. Microcirculation 1999; 6: 7-22.

[87] Takano T, Clish CB, Gronert K, et al. Neutrophil-mediated changes in vascular permeability are inhibited by topical application of aspirin-triggered 15-epi-lipoxin A4 and novel lipoxin B4 stable analogues. J Clin Invest 1998; 101: 819-26.

[88] Eltzschig HK, Ibla JC, Furuta GT, et al. Coordinated adenine nucleotide phosphohydrolysis and nucleoside signaling in posthypoxic endothelium: role of ectonucleotidases and adenosine $\mathrm{A}_{2 \mathrm{~B}}$ receptors. J Ex Med 2003; 198: 783-96.

[89] Eltzschig HK, Rivera-Nieves J, Colgan SP. Targeting the A2B adenosine receptor during gastrointestinal ischemia and inflammation. Expert Opin Ther Targets 2009; 13: 1267-77.

[90] Weissmueller T, Eltzschig HK, Colgan SP. Dynamic purine signalling and metabolism during neutrophil-endothelial interactions. . Purinergic Signalling 2005; 1: 229-39.

[91] Comerford KM, Wallace TJ, Karhausen J, et al. Hypoxia-inducible factor-1-dependent regulation of the multidrug resistance $(M D R l)$ gene. Cancer research 2002; 62: 3387-94.

[92] Cummins EP, Seeballuck F, Keely SJ, et al. The hydroxylase inhibitor dimethyloxalylglycine is protective in a murine model of colitis. Gastroenterology 2008; 134: 156-65.

[93] Han IO, Kim HS, Kim HC, et al. Synergistic expression of inducible nitric oxide synthase by phorbol ester and interferon-gamma is mediated through NF-kappaB and ERK in microglial cells. J Neurosci Res 2003; 73: 659-69.

[94] Morote-Garcia JC, Rosenberger P, Nivillac NM, et al. Hypoxiainducible factor-dependent repression of equilibrative nucleoside transporter 2 attenuates mucosal inflammation during intestinal hypoxia. Gastroenterology 2009; 136: 607-18.

[95] Robinson A, Keely S, Karhausen J, et al. Mucosal protection by hypoxia-inducible factor prolyl hydroxylase inhibition. Gastroenterology 2008; 134: 145-55.

[96] Shah YM, Ito S, Morimura K, et al. Hypoxia-inducible factor augments experimental colitis through an MIF-dependent inflammatory signaling cascade. Gastroenterology 2008; 134: 2036-48.

[97] Giatromanolaki A, Sivridis E, Maltezos E, et al. Hypoxia inducible factor 1alpha and 2alpha overexpression in inflammatory bowel disease. J Clin Pathol 2003; 56: 209-13.

[98] Mariani F, Sena P, Marzona L, et al. Cyclooxygenase-2 and Hypoxia-Inducible Factor-1alpha protein expression is related to inflammation, and up-regulated since the early steps of colorectal carcinogenesis. Cancer Lett 2009; 279: 221-9.

[99] Matthijsen RA, Derikx JP, Kuipers D, et al. Enterocyte shedding and epithelial lining repair following ischemia of the human small intestine attenuate inflammation. PLoS One 2009; 4: e7045.

[100] Louis NA, Hamilton KE, Canny G, et al. Selective induction of mucin-3 by hypoxia in intestinal epithelia. J Cell Biochem 2006; 99: 1616-27.

[101] Kaelin WG, Jr., Ratcliffe PJ. Oxygen sensing by metazoans: the central role of the HIF hydroxylase pathway. Mol Cell 2008; 30: 393-402.

[102] Cummins EP, Berra E, Comerford KM, et al. Prolyl hydroxylase-1 negatively regulates IkappaB kinase-beta, giving insight into hypoxia-induced NFkappaB activity. Proc Natl Acad Sci USA 2006; 103: 18154-9. 
[103] Aragones J, Schneider M, Van Geyte K, et al. Deficiency or inhibition of oxygen sensor Phd1 induces hypoxia tolerance by reprogramming basal metabolism. Nat Genet 2008; 40: 170-80.

[104] Schneider M, Van Geyte K, Fraisl P, et al. Loss or Silencing of the PHD1 Prolyl Hydroxylase Protects Livers of Mice Against Ischemia/ Reperfusion Injury. Gastroenterology 2009; 138: 1143-54.

[105] Mazzone M, Dettori D, Leite de Oliveira R, et al. Heterozygous deficiency of PHD2 restores tumor oxygenation and inhibits metastasis via endothelial normalization. Cell 2009; 136: 839-51.

[106] Ozolins TR, Fisher TS, Nadeau DM, et al. Defects in embryonic development of EGLN1/PHD2 knockdown transgenic mice are associated with induction of Igfbp in the placenta. Biochem Biophys Res Commun 2009; 390: 372-6.

[107] Bishop T, Gallagher D, Pascual A, et al. Abnormal sympathoadrenal development and systemic hypotension in PHD3-/- mice. Mol Cell Biol 2008; 28: 3386-400.

[108] Mole DR, Schlemminger I, McNeill LA, et al. 2-oxoglutarate analogue inhibitors of HIF prolyl hydroxylase. Bioorg Med Chem Lett 2003; 13: 2677-80.
[109] Masson N, Ratcliffe PJ. HIF prolyl and asparaginyl hydroxylases in the biological response to intracellular O(2) levels. J Cell Sci 2003 116: 3041-9.

[110] Schofield CJ, Ratcliffe PJ. Oxygen sensing by HIF hydroxylases. Nat Rev Mol Cell Biol 2004; 5: 343-54.

[111] Bruick RK. Oxygen sensing in the hypoxic response pathway: regulation of the hypoxia-inducible transcription factor. Genes Dev 2003; 17: 2614-23.

[112] Nwogu JI, Geenen D, Bean M, et al. Inhibition of collagen synthesis with prolyl 4-hydroxylase inhibitor improves left ventricular function and alters the pattern of left ventricular dilatation after myocardial infarction. Circulation 2001; 104: 2216-21.

[113] Schlemminger I, Mole DR, McNeill LA, et al. Analogues of dealanylalahopcin are inhibitors of human HIF prolyl hydroxylases. Bioorg Med Chem Lett 2003; 13: 1451-4.

[114] Greten FR, Eckmann L, Greten TF, et al. IKKbeta links inflammation and tumorigenesis in a mouse model of colitis-associated cancer. Cell 2004; 118: 285-96.

(c) Kelly and Colgan; Licensee Bentham Open.

This is an open access article licensed under the terms of the Creative Commons Attribution Non-Commercial License (http://creativecommons.org/licenses/ by-nc/3.0/) which permits unrestricted, non-commercial use, distribution and reproduction in any medium, provided the work is properly cited. 